\title{
An estimate on the thickness of boundary layer for nonlinear evolution equations with damping and diffusion
}

\author{
Wenshu Zhou', Xulong Qin² and Xiaodan Wei ${ }^{3 *}$
}

\section{"Correspondence:}

weixiaodancat@126.com

${ }^{3}$ School of Computer Science,

Dalian Nationalities University,

Dalian, 116600, China

Full list of author information is

available at the end of the article

\begin{abstract}
The main purpose of this paper is to estimate the thickness of boundary layer for nonlinear evolution equations with damping and diffusion as the diffusion parameter $\beta$ goes to zero. We prove that the thickness of layer is of the order $O\left(\beta^{\gamma}\right)$ with $0<\gamma<1$, thus improving the corresponding result in (Ruan and Zhu in Discrete Contin. Dyn. Syst. 32(1) 331-352, 2012) where $0<\gamma<1 / 2$ is obtained.
\end{abstract}

MSC: $35 \mathrm{k} 50 ; 35 \mathrm{~B} 40 ; 76 \mathrm{~N} 20$

Keywords: nonlinear evolution equation; zero diffusion limit; boundary layer thicknes

\section{Introduction}

In this paper, we consider the nonlinear evolution equations with damping and diffusion:

$$
\left\{\begin{array}{l}
\psi_{t}^{\beta}=-(\sigma-\alpha) \psi^{\beta}-\sigma \theta_{x}^{\beta}+\alpha \psi_{x x}^{\beta}, \\
\theta_{t}^{\beta}=-(1-\beta) \theta^{\beta}+\mu \beta \psi_{x}^{\beta}+2 \psi^{\beta} \theta_{x}^{\beta}+\beta \theta_{x x}^{\beta}, \quad 0<x<1, t>0,
\end{array}\right.
$$

with the initial-boundary conditions

$$
\begin{aligned}
& \left(\psi^{\beta}, \theta^{\beta}\right)(x, 0)=\left(\psi_{0}, \theta_{0}\right)(x), \quad 0 \leq x \leq 1, \\
& \left(\psi^{\beta}, \theta^{\beta}\right)(1, t)=\left(\psi^{\beta}, \theta^{\beta}\right)(0, t)=(0,0), \quad t \geq 0,
\end{aligned}
$$

where $\sigma, \alpha, \beta$, and $\mu$ are positive constants with $\alpha<\sigma$ and $0<\beta<1$. The corresponding problem of zero diffusion limit as $\beta \rightarrow 0$ is the following:

$$
\left\{\begin{array}{l}
\psi_{t}^{0}=-(\sigma-\alpha) \psi^{0}-\sigma \theta_{x}^{0}+\alpha \psi_{x x}^{0}, \\
\theta_{t}^{0}=-\theta^{0}+2 \psi^{0} \theta_{x}^{0}, \quad 0<x<1, t>0,
\end{array}\right.
$$

with the initial-boundary conditions

$$
\begin{aligned}
& \left(\psi^{0}, \theta^{0}\right)(x, 0)=\left(\psi_{0}, \theta_{0}\right)(x), \quad 0 \leq x \leq 1, \\
& \psi^{0}(1, t)=\psi^{0}(0, t)=0, \quad t \geq 0 .
\end{aligned}
$$

(c) 2016 Zhou et al. This article is distributed under the terms of the Creative Commons Attribution 4.0 International License (http://creativecommons.org/licenses/by/4.0/), which permits unrestricted use, distribution, and reproduction in any medium, provided you give appropriate credit to the original author(s) and the source, provide a link to the Creative Commons license, and indicate if changes were made. 
The system (1.1) was originally proposed by Hsieh in [2] to observe the nonlinear interaction between ellipticity and dissipation. In [3], Hsieh et al. established a link between this interaction and chaos. We also refer to $[4,5]$ for the physical background of (1.1). Some similar problems were studied in $[6,7]$ and the references therein.

Our main purpose is to estimate the thickness of boundary layer for problem (1.1)-(1.2) as $\beta \rightarrow 0$. Before stating the main result, we first recall the concept of BL-thickness in the sprit of [8].

Definition 1.1 A function $\delta(\beta)$ is called a BL-thickness for problem (1.1)-(1.2) with vanishing diffusion if $\delta(\beta) \downarrow 0$ as $\beta \downarrow 0$, and

$$
\begin{aligned}
& \lim _{\beta \rightarrow 0}\left\|\psi^{\beta}-\psi^{0}\right\|_{L^{\infty}\left(0, T ; L^{\infty}[0,1]\right)}=0, \\
& \lim _{\beta \rightarrow 0}\left\|\theta^{\beta}-\theta^{0}\right\|_{L^{\infty}\left(0, T ; L^{\infty}[\delta, 1-\delta]\right)}=0, \\
& \inf _{\beta \rightarrow 0}\left\|\theta^{\beta}-\theta^{0}\right\|_{L^{\infty}\left(0, T ; L^{\infty}[0,1]\right)}>0,
\end{aligned}
$$

for any $T>0$, where $\left(\psi^{\beta}, \theta^{\beta}\right)\left(\right.$ resp. $\left.\left(\psi^{0}, \theta^{0}\right)\right)$ is the solution for problem (1.1)-(1.2) (resp. problem (1.3)-(1.4)).

The theory of boundary layers is one of the most fundamental and important issues in fluid dynamics $(c f .[9,10])$ since the seminal work by Prandtl in 1904 . There are a number of papers dedicated to the questions of boundary layers for the Navier-Stokes equations; see for instance [8,11-17] and the references therein. Moreover, the boundary layer problem also arises in the theory of hyperbolic systems when parabolic equations with small viscosity are applied as perturbations; see for instance [18-23] and the references therein.

Recently, Ruan and Zhu [1], Theorem 1.3, discussed the existence and zero diffusion limit for problem (1.1)-(1.2), and proved that the thickness of boundary layer is of the order $O\left(\beta^{\gamma}\right)$ with $0<\gamma<1 / 2$ if $\frac{(\sigma+\mu \beta)^{2}}{4(1-\beta)}<\alpha<\sigma$ and if the initial data satisfy $\psi_{0} \in H^{2}([0,1]), \theta_{0} \in H^{3}([0,1]),\left(\psi_{0}, \theta_{0}\right)(1)=\left(\psi_{0}, \theta_{0}\right)(0)=(0,0)$, and $\left\|\left(\psi_{0}, \theta_{0}\right)\right\|_{2}$ is sufficiently small. Here $H^{l}([0,1])$ denotes the usual $l$ th order Sobolev space with the norm $\|f\|_{l}=\left(\sum_{i=0}^{l} \int_{0}^{1}\left|\partial_{x}^{i} f\right|^{2} d x\right)^{1 / 2}$. In the present paper, we improve the result by extending the range of $\gamma$ to $(0,1)$. Our main result can be stated as follows.

Theorem 1.2 Let $0<\beta<1$ and $\frac{(\sigma+\mu \beta)^{2}}{4(1-\beta)}<\alpha<\sigma$. Assume that the initial data satisfy $\psi_{0} \in H^{2}([0,1]), \theta_{0} \in H^{3}([0,1]),\left(\psi_{0}, \theta_{0}\right)(1)=\left(\psi_{0}, \theta_{0}\right)(0)=(0,0)$, and $\left\|\left(\psi_{0}, \theta_{0}\right)\right\|_{2}$ is sufficiently small. Then any function $\delta(\beta)$ satisfying $\delta(\beta) \downarrow 0$ and $\frac{\beta}{\delta(\beta)} \rightarrow 0$ as $\beta \downarrow 0$ is a BLthickness such that

$$
\left\|\theta^{\beta}-\theta^{0}\right\|_{L^{\infty}\left(0, T ; L^{\infty}[\delta, 1-\delta]\right)} \leq C \sqrt{\frac{\beta}{\delta}}, \quad \forall \delta \in(0,1 / 2),
$$

where $T>0$, and $C$ is a positive constant independent of $\beta$ and $\delta$.

The proof of Theorem 1.2 will be given in the next section.

\section{Proof of Theorem 1.2}

To prove Theorem 1.2, we need the following result, which can be found in [1], Lemmas 2.2, 2.4, 2.5 and 3.1. 
Lemma 2.1 Let the assumptions of Theorem 1.2 hold. Then there exists a positive constant independent of $\beta$ such that

$$
\begin{aligned}
& \int_{0}^{1}\left[\left(\psi_{x}^{\beta}\right)^{2}+\left(\theta_{x}^{\beta}\right)^{2}+\left(\psi_{x x}^{\beta}\right)^{2}+\beta\left(\theta_{x x}^{\beta}\right)^{2}\right] d x \leq C, \\
& \int_{0}^{1}\left[\left(\psi_{x}^{0}\right)^{2}+\left(\theta_{x}^{0}\right)^{2}+\left(\theta_{x x}^{0}\right)^{2}+\left(\psi_{x x}^{0}\right)^{2}\right] d x \leq C
\end{aligned}
$$

and

$$
\int_{0}^{1}\left[\left(\psi^{\beta}-\psi^{0}\right)^{2}+\left(\theta^{\beta}-\theta^{0}\right)^{2}\right] d x+\int_{0}^{T} \int_{0}^{1}\left(\psi^{\beta}-\psi^{0}\right)_{x}^{2} d x d t \leq C \beta .
$$

Proof of Theorem 1.2 It suffices to prove (1.5). Set

$$
u^{\beta}=\psi^{\beta}-\psi^{0}, \quad v^{\beta}=\theta^{\beta}-\theta^{0} .
$$

Then it follows from the equation of $\theta^{\beta}$ that

$$
v_{t}^{\beta}=-(1-\beta) v^{\beta}+2 \psi^{\beta} v_{x}^{\beta}+2 u^{\beta} \theta_{x}^{0}+\beta v_{x x}^{\beta}+\beta\left(\mu \psi_{x}^{\beta}+\theta^{0}+\theta_{x x}^{0}\right) .
$$

Differentiating the equation, we see that $z:=v_{x}^{\beta}$ satisfies

$$
z_{t}=-(1-\beta) z+2\left(\psi^{\beta} z\right)_{x}+2\left(u^{\beta} \theta_{x}^{0}\right)_{x}+\beta z_{x x}+\beta\left(\mu \psi_{x x}^{\beta}+\theta_{x}^{0}+\theta_{x x x}^{0}\right) .
$$

Denote $\varphi_{\varepsilon}$ for $\varepsilon \in(0,1)$ and $\xi_{\delta}$ for $\delta \in(0,1 / 2)$ by

$$
\varphi_{\varepsilon}(s)=\sqrt{s^{2}+\varepsilon^{2}}, \quad \xi_{\delta}(x)= \begin{cases}x, & 0 \leq x \leq \delta \\ \delta, & \delta \leq x \leq 1-\delta \\ 1-x, & 1-\delta \leq x \leq 1\end{cases}
$$

It is easy to check that $\varphi_{\varepsilon}$ satisfies

$$
\left\{\begin{array}{l}
|s| \leq\left|\varphi_{\varepsilon}(s)\right| \leq|s|+1 \\
\left|\varphi_{\varepsilon}^{\prime}(s)\right| \leq 1, \quad 0 \leq s \varphi_{\varepsilon}^{\prime}(s) \leq \varphi_{\varepsilon}(s) \\
\varphi_{\varepsilon}^{\prime \prime}(s) \geq 0, \quad s^{2} \varphi_{\varepsilon}^{\prime \prime}(s) \geq 0
\end{array}\right.
$$

and $\xi_{\delta}$ satisfies

$$
0 \leq \xi_{\delta} \leq \delta, \quad \xi_{\delta}(1)=\xi_{\delta}(0)=0
$$

Multiplying (2.4) by $\varphi_{\varepsilon}^{\prime}(z) \xi_{\delta}$ and integrating it over $(0,1) \times(0, t)$, we have

$$
\begin{aligned}
& \int_{0}^{1} \varphi_{\varepsilon}(z) \xi_{\delta} d x-\varepsilon \int_{0}^{1} \xi_{\delta} d x \\
& \quad=-(1-\beta) \int_{0}^{t} \int_{0}^{1} z \varphi_{\varepsilon}^{\prime}(z) \xi_{\delta} d x d \tau+2 \int_{0}^{t} \int_{0}^{1}\left(\psi^{\beta} z\right)_{x} \varphi_{\varepsilon}^{\prime}(z) \xi_{\delta} d x d \tau
\end{aligned}
$$




$$
\begin{aligned}
& +2 \int_{0}^{t} \int_{0}^{1}\left(u^{\beta} \theta_{x}^{0}\right)_{x} \varphi_{\varepsilon}^{\prime}(z) \xi_{\delta} d x d \tau+\beta \int_{0}^{t} \int_{0}^{1} z_{x x} \varphi_{\varepsilon}^{\prime}(z) \xi_{\delta} d x d \tau \\
& +\beta \int_{0}^{t} \int_{0}^{1} \varphi_{\varepsilon}^{\prime}(z) \xi_{\delta}\left(\mu \psi_{x x}^{\beta}+\theta_{x}^{0}+\theta_{x x x}^{0}\right) d x d \tau=: \sum_{i=1}^{5} E_{j} .
\end{aligned}
$$

Next we estimate $E_{i}(i=1,2,3,4,5)$. From $0 \leq s \varphi_{\varepsilon}^{\prime}(s) \leq \varphi_{\varepsilon}(s)$, we have

$$
E_{1} \leq \int_{0}^{t} \int_{0}^{1} \varphi_{\varepsilon}(z) \xi_{\delta} d x d \tau
$$

To estimate $E_{2}$, we note, using integration by parts,

$$
\begin{aligned}
E_{2}= & 2 \int_{0}^{t} \int_{0}^{1} \psi_{x}^{\beta} z \varphi_{\varepsilon}^{\prime}(z) \xi_{\delta} d x d \tau+2 \int_{0}^{t} \int_{0}^{1} \psi^{\beta} z_{x} \varphi_{\varepsilon}^{\prime}(z) \xi_{\delta} d x d \tau \\
= & 2 \int_{0}^{t} \int_{0}^{1} \psi_{x}^{\beta} z \varphi_{\varepsilon}^{\prime}(z) \xi_{\delta} d x d \tau-2 \int_{0}^{t} \int_{0}^{1} \varphi_{\varepsilon}(z) \psi_{x}^{\beta} \xi_{\delta} d x d \tau \\
& -2 \int_{0}^{t} \int_{0}^{1} \varphi_{\varepsilon}(z) \psi^{\beta} \xi_{\delta}^{\prime} d x d \tau \\
=: & E_{2}^{1}+E_{2}^{2}+E_{2}^{3} .
\end{aligned}
$$

By (2.1) and the embedding $W^{1,1}[0,1] \hookrightarrow L^{\infty}[0,1]$, we have

$$
\left|\psi_{x}^{\beta}(x, t)\right| \leq \int_{0}^{1}\left|\psi_{x}^{\beta}\right| d x+\int_{0}^{1}\left|\psi_{x x}^{\beta}\right| d x \leq C,
$$

where $C$ denotes the generic positive constant independent of $\beta, \delta$, and $\varepsilon$, so

$$
\begin{aligned}
& \left|\psi^{\beta}(x, t)\right| \leq \int_{0}^{x}\left|\psi_{y}^{\beta}(y, t)\right| d y \leq C x \leq C \xi_{\delta}(x), \quad \forall x \in[0, \delta], \\
& \left|\psi^{\beta}(x, t)\right| \leq \int_{x}^{1}\left|\psi_{y}^{\beta}(y, t)\right| d y \leq C(1-x) \leq C \xi_{\delta}(x), \quad \forall x \in[1-\delta, 1] .
\end{aligned}
$$

By $0 \leq s \varphi_{\varepsilon}^{\prime}(s) \leq \varphi_{\varepsilon}(s)$ and (2.8), we obtain

$$
E_{2}^{1}+E_{2}^{2} \leq C \int_{0}^{t} \int_{0}^{1} \varphi_{\varepsilon}(z) \xi_{\delta} d x d \tau
$$

By the definition of $\xi_{\delta}$ and (2.9), we have

$$
\begin{aligned}
E_{2}^{3} & =-2 \int_{0}^{t} \int_{0}^{\delta} \varphi_{\varepsilon}(z) \psi^{\beta} d x d \tau+2 \int_{0}^{t} \int_{1-\delta}^{1} \varphi_{\varepsilon}(z) \psi^{\beta} d x d \tau \\
& \leq C \int_{0}^{t} \int_{0}^{\delta} \varphi_{\varepsilon}(z) \xi_{\delta} d x d \tau+C \int_{0}^{t} \int_{1-\delta}^{1} \varphi_{\varepsilon}(z) \xi_{\delta} d x d \tau \\
& \leq C \int_{0}^{t} \int_{0}^{1} \varphi_{\varepsilon}(z) \xi_{\delta} d x d \tau .
\end{aligned}
$$

Thus

$$
E_{2} \leq C \int_{0}^{t} \int_{0}^{1} \varphi_{\varepsilon}(z) \xi_{\delta} d x d \tau
$$


Using integration by parts and noticing $\varphi_{\varepsilon}^{\prime \prime} \geq 0$ and $\left|\varphi_{\varepsilon}^{\prime}\right| \leq 1$, we have

$$
\begin{aligned}
E_{4} & =-\beta \int_{0}^{t} \int_{0}^{1} z_{x}^{2} \varphi_{\varepsilon}^{\prime \prime}(z) \xi_{\delta} d x d \tau-\beta \int_{0}^{t} \int_{0}^{1} z_{x} \varphi_{\varepsilon}^{\prime}(z) \xi_{\delta}^{\prime} d x d \tau \\
& \leq-\beta \int_{0}^{t} \int_{0}^{1} z_{x} \varphi_{\varepsilon}^{\prime}(z) \xi_{\delta}^{\prime} d x d \tau \\
& =-\beta \int_{0}^{t} \int_{0}^{\delta} z_{x} \varphi_{\varepsilon}^{\prime}(z) d x d \tau+\beta \int_{0}^{t} \int_{1-\delta}^{1} z_{x} \varphi_{\varepsilon}^{\prime}(z) d x d \tau \\
& \leq \beta\left(\int_{0}^{t} \int_{0}^{\delta}\left|z_{x}\right| d x d \tau+\int_{0}^{t} \int_{1-\delta}^{1}\left|z_{x}\right| d x d \tau\right)
\end{aligned}
$$

and, by Hölder's inequality and (2.1), we obtain

$$
\begin{aligned}
E_{4} & \leq C \beta \delta^{1 / 2}\left[\left(\int_{0}^{t} \int_{0}^{\delta}\left|z_{x}\right|^{2} d x d \tau\right)^{1 / 2}+\left(\int_{0}^{t} \int_{1-\delta}^{1}\left|z_{x}\right|^{2} d x d \tau\right)^{1 / 2}\right] \\
& \leq C \beta^{1 / 2} \delta^{1 / 2}
\end{aligned}
$$

By $\left|\varphi_{\varepsilon}^{\prime}\right| \leq 1,0 \leq \xi_{\delta} \leq \delta$, Hölder's inequality, (2.2), and (2.3), we have

$$
\begin{aligned}
E_{3}= & 2 \int_{0}^{t} \int_{0}^{1} u_{x}^{\beta} \theta_{x}^{0} \varphi_{\varepsilon}^{\prime}(z) \xi_{\delta} d x d \tau+2 \int_{0}^{t} \int_{0}^{1} u^{\beta} \theta_{x x}^{0} \varphi_{\varepsilon}^{\prime}(z) \xi_{\delta} d x d \tau \\
\leq & C \delta\left(\int_{0}^{t} \int_{0}^{1}\left(u_{x}^{\beta}\right)^{2} d x d \tau\right)^{1 / 2}\left(\int_{0}^{t} \int_{0}^{1}\left(\theta_{x}^{0}\right)^{2} d x d \tau\right)^{1 / 2} \\
& +C \delta\left(\int_{0}^{t} \int_{0}^{1}\left(u^{\beta}\right)^{2} d x d \tau\right)^{1 / 2}\left(\int_{0}^{t} \int_{0}^{1}\left(\theta_{x x}^{0}\right)^{2} d x d \tau\right)^{1 / 2} \\
\leq & C \delta \beta^{1 / 2} .
\end{aligned}
$$

Finally, we estimate $E_{5}$. By $\left|\varphi_{\varepsilon}^{\prime}\right| \leq 1,0 \leq \xi_{\delta} \leq \delta$, and Lemma 2.1, we have

$$
\begin{aligned}
E_{5} & \leq C \beta \delta \int_{0}^{t} \int_{0}^{1}\left(\left|\psi_{x x}^{\beta}\right|+\left|\theta_{x}^{0}\right|+\left|\theta_{x x x}^{0}\right|\right) d x d \tau \\
& \leq C \beta \delta .
\end{aligned}
$$

Combining (2.6), (2.12)-(2.15) with (2.5) and noticing

$$
\varepsilon \int_{0}^{1} \xi_{\delta} d x \leq \varepsilon \delta
$$

we obtain

$$
\int_{0}^{1} \varphi_{\varepsilon}(z) \xi_{\delta} d x \leq C \int_{0}^{t} \int_{0}^{1} \varphi_{\varepsilon}(z) \xi_{\delta} d x d \tau+\varepsilon \delta+C \beta^{1 / 2} \delta^{1 / 2}
$$

so an application of Gronwall's inequality leads to

$$
\int_{0}^{1} \varphi_{\varepsilon}(z) \xi_{\delta} d x \leq C\left(\varepsilon \delta+\beta^{1 / 2} \delta^{1 / 2}\right)
$$


From this and the definition of $\xi_{\delta}$ and $|z| \leq \varphi_{\varepsilon}(z)$, we obtain

$$
\int_{\delta}^{1-\delta}|z| d x \leq C\left(\varepsilon+\sqrt{\frac{\beta}{\delta}}\right) .
$$

Letting $\varepsilon \rightarrow 0$ yields

$$
\int_{\delta}^{1-\delta}|z| d x \leq C \sqrt{\frac{\beta}{\delta}}
$$

From (2.3), (2.16), and the embedding $W^{1,1}([\delta, 1-\delta]) \hookrightarrow L^{\infty}([\delta, 1-\delta])$ it follows that

$$
\begin{aligned}
\left\|\left(\theta^{\beta}-\theta^{0}\right)(\cdot, t)\right\|_{L^{\infty}[\delta, 1-\delta]} & \leq \int_{0}^{1}\left|\theta^{\beta}-\theta^{0}\right| d x+\int_{\delta}^{1-\delta}|z| d x \\
& \leq C \sqrt{\frac{\beta}{\delta}} .
\end{aligned}
$$

Thus (1.5) is proved, and the proof is complete.

\section{Competing interests}

The authors declare that they have no competing interests.

\section{Authors' contributions}

All authors contributed to each part of this work equally.

\section{Author details}

${ }^{1}$ Department of Mathematics, Dalian Nationalities University, Dalian, 116600, China. ${ }^{2}$ Department of Mathematics, Sun-Yat University, Guangzhou, 510275, China. ${ }^{3}$ School of Computer Science, Dalian Nationalities University, Dalian, 116600, China.

\section{Acknowledgements}

We would like to thank the referees for their important comments which have improved our paper. The research was supported in part by the NSFC (grants 11571062, 11571380), the Program for Liaoning Excellent Talents in University (grant LJQ2013124) and the Fundamental Research Fund for the Central Universities (grant DC201502050202).

Received: 1 June 2016 Accepted: 30 September 2016 Published online: 12 October 2016

\section{References}

1. Ruan, LZ, Zhu, CJ: Boundary layer for nonlinear evolution equations with damping and diffusion. Discrete Contin. Dyn. Syst. 32(1), 331-352 (2012)

2. Hsieh, DY: On partial differential equations related to Lorenz system. J. Math. Phys. 28, 1589-1597 (1987)

3. Hsieh, DY, Tang, SQ, Wang, YP, Wu, LX: Dissipative nonlinear evolution equations and chaos. Stud. Appl. Math. 101(3), 233-266 (1998)

4. Keefe, LR: Dynamics of perturbed wavetrain solutions to the Ginzberg-Landau equation. Stud. Appl. Math. 73, 91-152 (1985)

5. Kuramoto, Y, Tsuzuki, T: On the formation of dissipative structures in reaction-diffusion systems. Prog. Theor. Phys. 54, 687-699 (1975)

6. Peng, HY, Ruan, LZ, Xiang, JL: A note on boundary layer of a nonlinear evolution system with damping and diffusions. J. Math. Anal. Appl. 426, 1099-1129 (2015)

7. Peng, Y: Boundary layer and vanishing diffusion limit for nonlinear evolution equations. Acta Math. Sci. 34(4), 1271-1286 (2014)

8. Frid, H, Shelukhin, V: Boundary layers for the Navier-Stokes equations of compressible fluids. Commun. Math. Phys. 208, 309-330 (1999)

9. Landau, LD, Lifshitz, EM: Fluid Mechanics, 2nd edn. Pergamon Press, Oxford (1987)

10. Schlichting, H: Boundary Layer Theory, 7th edn. McGraw-Hill Company, London-New York (1979)

11. E, WN: Boundary layer theory and the zero-viscosity limit of the Navier-Stokes equation. Acta Math. Sin. Engl. Ser. 16(2), 207-218 (2000)

12. File, PC: Considerations regarding the mathematical basis for Prandtl's boundary layer theory. Arch. Ration. Mech. Anal. 28, 184-216 (1968)

13. Temam, $R$, Wang, $X$ : Boundary layers associated with incompressible Navier-Stokes equations: the noncharacteristic boundary case. J. Differ. Equ. 179, 647-686 (2002)

14. Wang, YG, Xin, ZP: Zero-viscosity limit of the linearized compressible Navier-Stokes equations with highly oscillatory forces in the half-plane. SIAM J. Math. Anal. 37, 1256-1298 (2005) 
15. Jiang, S, Zhang, JW: Boundary layers for the Navier-Stokes equations of compressible heat-conducting flows with cylindrical symmetry. SIAM J. Math. Anal. 41, 237-268 (2009)

16. Yao, L, Zhang, T, Zhu, CJ: Boundary layers for compressible Navier-Stokes equations with density-dependent viscosity and cylindrical symmetry. Ann. Inst. Henri Poincaré, Anal. Non Linéaire 28, 677-709 (2011)

17. Qin, XL, Yang, T, Yao, ZA, Zhou, WS: Vanishing shear viscosity and boundary layer for the Navier-Stokes equations with cylindrical symmetry. Arch. Ration. Mech. Anal. 216, 1049-1086 (2015)

18. Frid, $\mathrm{H}$, Shelukhin, V: Boundary layers in parabolic perturbations of scalar conservation laws. Z. Angew. Math. Phys. 55, 420-434 (2004)

19. Grenier, E, Guès, O: Boundary layers for viscous perturbations of noncharacteristic quasi-linear hyperbolic problems. J. Differ. Equ. 143, 110-146 (1998)

20. Grenier, E, Rousset, F: Stability of one-dimensional boundary layers by using Green's functions. Commun. Pure Appl. Math. 54(11), 1343-1385 (2001)

21. Serre, D, Zumbrun, K: Boundary layer stability in real vanishing viscosity limit. Commun. Math. Phys. 221, 267-292 (2001)

22. Shelukhin, V: A shock layer in parabolic perturbations of a scalar conservation law. Proc. Edinb. Math. Soc. 46, 315-328 (2003)

23. Xin, ZP: Viscous boundary layers and their stability (I). J. Partial Differ. Equ. 11, 97-124 (1998)

\section{Submit your manuscript to a SpringerOpen ${ }^{\ominus}$ journal and benefit from:}

- Convenient online submission

Rigorous peer review

- Immediate publication on acceptance

- Open access: articles freely available online

- High visibility within the field

- Retaining the copyright to your article 